\title{
Engineering Challenges in W7-X: lessons learned and status for the second operation phase
}

\author{
H.-S, Bosch. , T. Andreeva, R. Brakel, T. Bräuer, D. Hartmann, A. Holtz, T. Klinger, H. Laqua, \\ M. Nagel, D. Naujoks, K. Risse, A. Spring, T. Sunn Pedersen, T. Rummel, P. van Eeten, A. Werner, \\ R. Wolf and the W7-X Team
}

\begin{abstract}
In 2015 the optimized stellarator Wendelstein 7-X stellarator (W7-X) started with operation. The main objective of $\mathrm{W} 7-\mathrm{X}$ is the demonstration of the integrated reactor potential of the optimized stellarator line. An important element of this mission is the achievement of high heating-power and high confinement in steady-state operation. The approach to this mission is following three steps. First plasmas were produced in a limiter configuration (OP 1.1), then a test divertor unit is being installed (TDU) for the next campaign, OP 1.2, before the full steady state capability will be achieved implementing active cooling of all in-vessel components and a steady state high heat flux divertor. In December 2015, the first helium plasma was generated using Electron Cyclotron Resonance Heating (ECRH), in February 2016 the working gas was switched to hydrogen. The first operation phase (OP 1.1) was successfully finished in March 2016. At the end of OP 1.1 the discharge duration was close to 6 seconds, the limit for the integrated heating power was increased to $4 \mathrm{MJ}$ and electron temperatures of $\sim 10 \mathrm{keV}$ were achieved. Due to the low densities in the range of $10^{19} \mathrm{~cm}^{-3}$ and the pure electron heating by ECRH, the ion temperatures reached only $2 \mathrm{keV}$.

At present, $\mathrm{W} 7-\mathrm{X}$ is undergoing the next completion phase, including the installation of the test divertor unit, the installation of the carbon tiles on the inner plasma vessel wall, an upgrade of existing diagnostics and the installation of new diagnostics.

This paper discusses the first operational phase, lessons learned and implemented and the status before the start of the second operational phase (OP 1.2).
\end{abstract}

Index Terms-Stellarator, Wendelstein 7-X, operation, heating, divertor

\section{INTRODUCTION}

Wendelstein 7-X (W7-X) is a modular advanced stellarator [1], which successfully went into operation in December 2015 at the Max-Planck-Institute for plasma physics in Greifswald, Germany [2]. The term modular stellarator refers to a generalized stellarator configuration with nested magnetic surfaces created by a system of toroidally discrete coils, providing both toroidal and poloidal field components. The asdesigned coil shapes were modeled based on the optimized

\footnotetext{
"This work has been carried out within the framework of the EUROfusion Consortium and has received funding from the Euratom research and training programme 2014-2018 under grant agreement No 633053. The views and pinions expressed herein do not necessarily reflect those of the European Commission."
}

equilibrium properties [3]. This optimization included the high quality of vacuum magnetic surfaces, good finite beta equilibrium and magnetohydrodynamic (MHD)-stability properties as well as a substantial reduction of the neoclassical transport and bootstrap current. The mission of the W7-X project is to demonstrate the reactor potential of the optimized stellarator line.

W7-X, see figure 1, has following main parameters: an average major radius of $5.5 \mathrm{~m}$, an average minor plasma radius of $0.53 \mathrm{~m}$, a total weight of $725 \mathrm{t}$, a cold mass of $435 \mathrm{t}$, a plasma volume of $30 \mathrm{~m}^{3}$ and a magnetic induction on the axis of 2.5 $3 \mathrm{~T}$. It is planned to operate W7-X in steady-state mode (30 min discharges) [4].

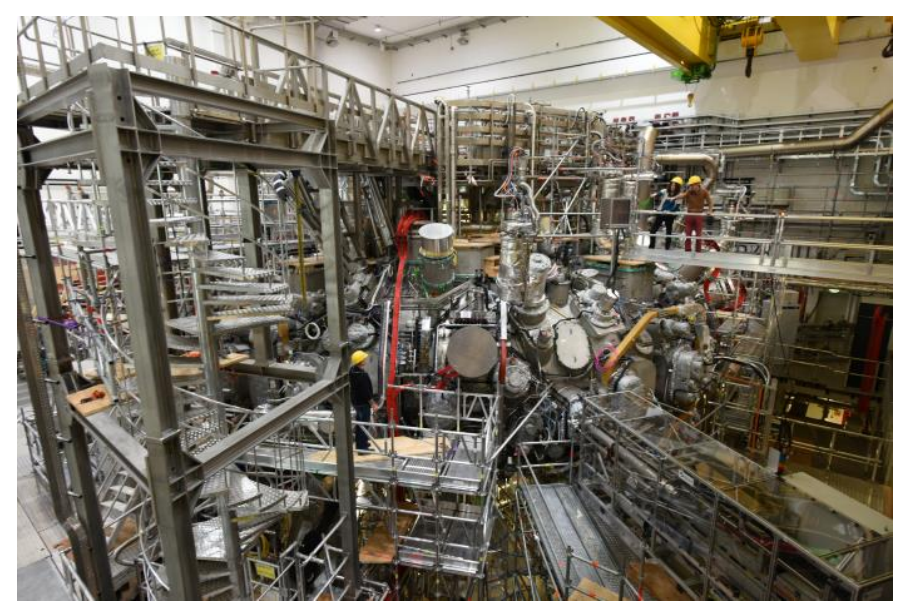

Fig. 1. Wendelstein 7-X in December 2015, a few days before the first plasma operation. On top of the device and along the backside wall, cable trays can be seen. On the left side in the picture, a bridge to the center of the torus, which also holds diagnostic components, is visible.

The approach to steady-state operation, however, is performed in different steps, as shown in table 1 . In the first operation phase, called OP 1.1, W7-X did not use a divertor, but was equipped only with 5 limiters on the inner wall. In the following completion phase CP $1.2 \mathrm{a}, \mathrm{W} 7-\mathrm{X}$ was equipped with

All authors are with the Max-Planck-institute for Plasma Physics in Greifswald, Germany (e-mail: author@ ipp.mpg.de). The main author can be contacted under: bosch@ipp.mpg.de 
an inertially cooled diverter (see chapter IV, section A). This inertially cooled grain graphite divertor is rather robust, and therefore allows developing divertor scenarios for the final steady-state, High Heat Flux Divertor to be installed in the CP 2 and to be operated in OP 2. Between the two TDU-phases OP 1.2a and 1.2b.an additional scraper element will be installed in one of the divertor modules for a test.

\begin{tabular}{c|c|l|l}
\hline start & \multicolumn{1}{|c|}{ end } & topic & description \\
\hline Apr 14 & Dec. 7, 2015 & Commissioning & $\begin{array}{l}\text { frst commissioning of the basic } \\
\text { device }\end{array}$ \\
\hline Dec. 7, 2015 & Mar 11, 2016 & OP 1.1 & $\begin{array}{l}\text { 1. operation phase with } 5 \text { graphite } \\
\text { limiters on the inner wall }\end{array}$ \\
\hline Mar 11, 2016 & May 5, 2017 & CP 1.2a & $\begin{array}{l}\text { Installation of the TDU, graphite } \\
\text { baffles and graphite tiles on the } \\
\text { wall }\end{array}$ \\
\hline May 5, 2017 & Dec. 8, 2017 & OP 1.2a* & $\begin{array}{l}\text { 2. operation phase with an inertially } \\
\text { cooled divertor (10 TDU) }\end{array}$ \\
\hline Dec. 8, 2017 & Apr 20, 2018 & CP 1.2b & $\begin{array}{l}\text { Installation of one TDU scraper } \\
\text { element }\end{array}$ \\
\hline Apr 20, 2018 & Oct. 19, 2018 & OP 1.2b* & $\begin{array}{l}\text { 3. operation phase with 10 TDU } \\
\text { and one scraper element }\end{array}$ \\
\hline Oct. 19, 2018 & Nov 19, 2020 & CP 2 & $\begin{array}{l}\text { Installation of the steady-state, } \\
\text { water-cooled divertor }\end{array}$ \\
\hline Nov 19, 2020 & t.b.d. & OP 2* & $\begin{array}{l}\text { 4. operation phase with the } \\
\text { transition to steady state operation }\end{array}$ \\
\hline
\end{tabular}

TABLE 1: List of the operation phases (OP) and completion phases (CP) of W7-X with a timeline and descriptions of the respective phases. All the OP phases designated with a $*$ include the respective device commissioning.

\section{ENGINEERING CHALLENGES DURING THE CONSTRUCTION OF W7-X}

During the fabrication of W7-X components and assembly process, many challenges came up, some of them were foreseen, others came up unexpectedly. One challenge, which was all the time in the center of the project, was the required accuracy of the numerically optimized magnetic field. This will be discussed in detail in the following section.

\section{A. Tolerances of the magnetic field}

The central component of the machine is a modular coil system, consisting of 50 non-planar and 20 planar superconducting coils, arranged in five identical modules and realizing a large variety of stellarator configurations, with a set of islands at the edge of the plasma forming a magnetic divertor. A significant part of the planned operational plasma scenarios in W7-X is rather sensitive to symmetry breaking perturbations which are resonant with the value of the rotational transform $1 / 2 \pi=1$ at the boundary and violate the toroidal periodicity of the magnetic field. As a consequence the W7-X island topology can be changed that would cause the redistribution of the power loads on the divertor plates. The reasons for the magnetic field perturbations are asymmetric deviations in coil shapes and positions resulting from manufacturing and assembly tolerances as well as from the deformations under the mechanical and electromagnetic loads.

Coil filament geometries are the basis for the analysis of W7$\mathrm{X}$ magnetic field and corresponding plasma properties. The magnetic field perturbation can be represented by the most relevant low-order components $\mathrm{B}_{\mathrm{m}, \mathrm{n}}$ of a poloidal-toroidal Fourier decomposition of the radial component of the magnetic field on a flux surface at the plasma edge. In case of W7-X operating scenarios these are the $\mathrm{B}_{11}, \mathrm{~B}_{22}, \mathrm{~B}_{33}$ and $\mathrm{B}_{44}$ components. The whole process of the magnet system construction was accompanied by the evaluation of the main components of the magnetic field perturbation based on the metrology supervision, which started with the manufacturing process of the winding packs (WPs) and was continued during the assembly at the Greifswald site [5].

During the WP fabrication the shape of the WPs was surveyed by measuring of positions of 768 points on the surface of the WP. For the 50 fabricated non-planar WPs, the absolute average deviations of the central filament position from the computer-aided-design (CAD) shape were $<3 \mathrm{~mm}$, and from the average as-built WP shape, evaluated for each WP type, < $2 \mathrm{~mm}$. For the planar WPs these numbers were 3.5 and $1.5 \mathrm{~mm}$, respectively [6]Then up to 12 reference marks per coil were defined within an uncertainty of less than $0.3 \mathrm{~mm}$ by a best-fit of the as-built winding package shape against the nominal CAD geometry. These reference marks were used to align and monitor coil positions in each assembly step including the final alignment of magnet system modules. The requirements on the alignment tolerances were of the order of $0.5 \mathrm{~mm}-1.5 \mathrm{~mm}$. On the whole, they were achieved in all alignment processes. A best-fit of latest measured reference mark co-ordinates against the as-manufactured coil reference mark co-ordinates gives a measure about the overall coil positioning errors caused by W7$\mathrm{X}$ assembly. Averaged over all reference marks of all coils the average assembly error for one assembly step was of the order of $1.2 \mathrm{~mm}$, whilst the maximum assembly error of all coils was less than $4.4 \mathrm{~mm}$. A comparison (without best-fit) of the latest measured reference mark co-ordinates with the as-manufacture coil reference mark co-ordinates gives the maximum displacement of a coil compared to its nominal position. In total no reference mark co-ordinate deviated more than $5.7 \mathrm{~mm}$ from its manufacture value, which is much smaller than the safety margin of gaps between components to avoid collisions. The main part of geometric measurements during coil assembly was performed using laser trackers. With the standard nominal $2 \sigma$ measurement uncertainty of the laser tracker of $0.01 \mathrm{~mm} / \mathrm{m} \pm$ $0.05 \mathrm{~mm}$, the practically achieved measurement uncertainty inside the experimental hall ranged between 0.15 and $0.5 \mathrm{~mm}$ depending on measurement distance and line of sight conditions.

Before placement of the assembled W7-X modules on the machine fundament, the new optimized target coordinate-set for the reference marks on the coils was calculated for each module in order to reduce magnetic field errors. It helped almost to eliminate the magnetic field perturbation accumulated during the manufacturing phase and previous assembly steps. The residual level of the magnetic field perturbations after the placement of the last machine module on the machine base was $0.34 \cdot 10^{-4}$ for the standard operating case and was in the same order of magnitude for all other reference magnetic configurations [7].

Several other sources of possible magnet system asymmetries were simulated with help of finite element (FE) models. Two $360^{\circ}$ FE global models of the Wendelstein 7-X 
magnet system were created with the ANSYS and ABAQUS codes $[10,11,12]$. By means of these Models deformations of all 70 superconducting coils under the dead-weight, cool-down and electromagnetic loads in different operation regimes were simulated. In addition the consequences of sequential loading of the machine base during W7-X assembly, the connection of adjacent modules to each other and the removal of temporary supports were modeled with the $360^{\circ}$ ABAQUS model. To cover the expected range of possible structural parameter variations of W7-X magnet system, the $72^{\circ}$ ANSYS Global Model was used. Taking into account results of all these simulations,

the final

level of the magnetic

field perturbation can be estimated as $(1.21 \pm 0.34$ $\pm 0.3) \cdot 10^{-4}$ [8], which is well below the

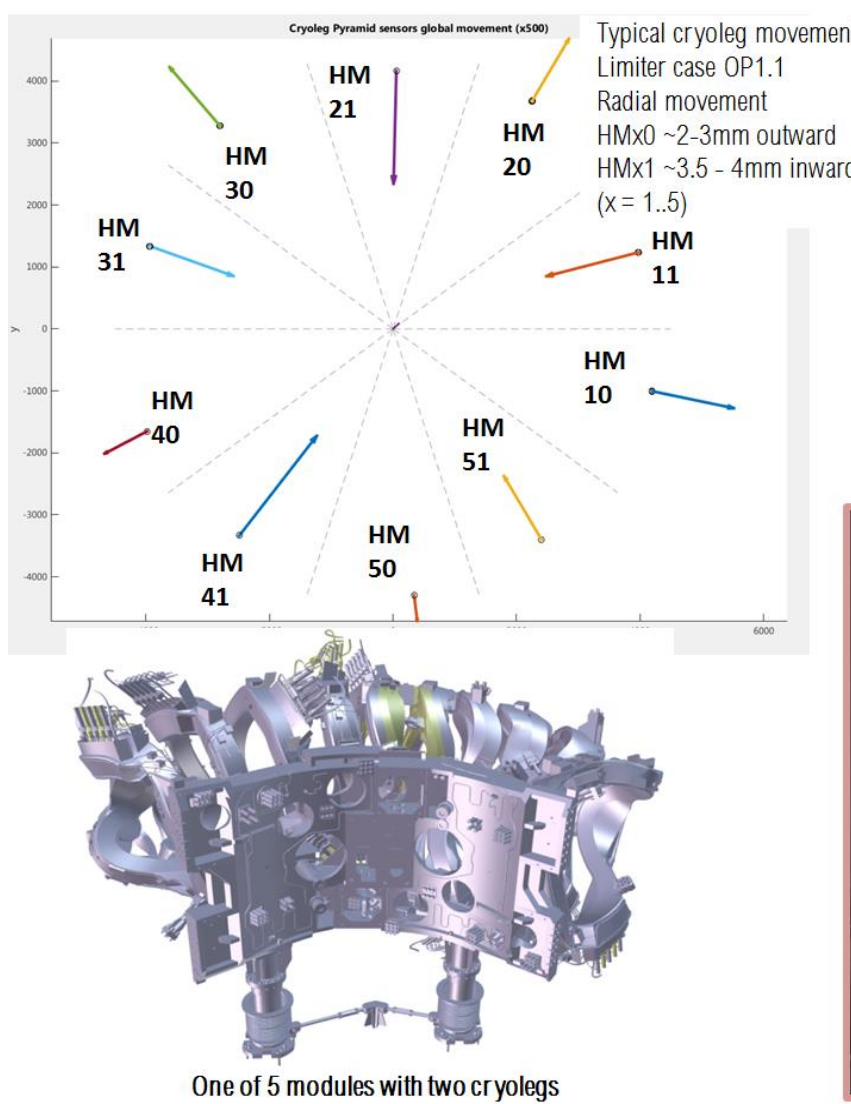

been commissioned up to $60 \%$ of the nominal values and operated on 9 experimental days [16].

\section{B. Cryo supply}

The cryogenic system consists of a helium refrigerator of equivalent cooling power of $7 \mathrm{~kW}$ at $4.2 \mathrm{~K}$, transfer lines with a valve box for helium distribution and the cold components inside the W7-X cryostat, such as magnets, structure and piping [17]. The two screw compressors of the refrigerator have a power consumption of 1.6 MW and provide the high pressure flow for the cold box. The refrigerator cools the thermal shield

Fig 2 Typical radial movement of cryoleg lower bearing: Measurement (upper left) vs. Prediction: Load case Limiter OP1.1 (upper right). Cryoleg location (lower left) and a typical measurement pyramid at the cryoleg lower bearing (lower right) are shown.

compensation capacities of the installed trim coils. In addition, the assessment of the magnet system deformation under electromagnetic forces due to the operation of the trim coils was performed. The corresponding error fields are of order of $0.25 \cdot 10^{-4}$ and can be compensated by a slight readjustment of the trim coil currents.

\section{FIRST OPERATION PHASE: LESSONS LEARNED}

\section{A. Magnet operation}

In first operational phase of W7-X, the superconducting magnet system was operated in a very stable way on 31 days with 35 load cycles, amounting to a time period of 183 hours. The precision of the current control was better that expected $[13,14]$.

The trim coils (copper coils, normally conducting) [15] had
[18] between $50-80 \mathrm{~K}$ and the high temperature current leads at $50 \mathrm{~K}$. Two cold circulators are used to push $500 \mathrm{~g} / \mathrm{s}$ of supercritical helium through the magnet and through the cooling channels of the cold structure. Temperatures below 4.2 $\mathrm{K}$ are achieved with a cold compressor that pumps a liquid helium bath down to a pressure of $660 \mathrm{hPa}$.

The cryogenic system worked reliable having only 9 trips during the period of OP1.1 [19]. Two third of the problems were caused by the cooling water system andsupply systems (e.g. power failure).

After one year of continuous operation during OP1.1 maintenance on the cryogenic system and especially on the refrigerator was essential. The work was done by the manufacturers for the more complex systems like the screw compressors with couplings, the electrical drives of the compressors, and the corresponding frequency converters. 
Some bearings of the cold circulators made noises during operation in OP1.1. So the bearings of all cold machines were checked by the manufacturer and replaced when needed. Also the safety valves in the refrigerator, on the helium distribution system and on the cryostat were dismounted and tested by an approved inspection body.

During operation of OP1.1 about $20 \%$ of the helium inventory of $13600 \mathrm{Nm} 3$ was lost. The main reason was the purging and cleaning procedure at the beginning of OP1.1 together with several regenerations of dryers and cold adsorber beds. In addition, a trip of the refrigerator occurred during the operation of W7-X due to an external power failure. That event led to an opening of safety valves and blow off of He-gas into the environment.

In OP1.1 four gas storage tanks were available with $250 \mathrm{Nm}^{3}$ each each which is at the limit to store a helium inventory of $2700 \mathrm{~kg}$. It turned out that for practical reason a fifth tank is needed to fulfill the following requirements:

- gas storage of the complete cold helium inventory (up to $2000 \mathrm{~kg}, 880 \mathrm{~m}^{3}$ )

- sufficient spare gas in case of unexpected helium loss during an experimental campaign (at least $700 \mathrm{~kg}, 120 \mathrm{~m}^{3}$ )

- one additional tank for loading and unloading the refrigerator at low pressure $\left(250 \mathrm{~m}^{3}\right)$

- one dedicated tank for quench gas recovery (is emptied during cool down)

Therefore it was decided to order a fifth gas storage tank.

The control system of the refrigerator was updated and extended to ensure a more stable operation of the control system (Windows 7 server with SIEMENS Process Control System named PCS-7 version 8.2). The system consists now of one server and one redundant server, two clients and in addition three virtual operation stations as clients. Face plates of the control system were modified to ease the use of the complex control program for the operators. Also online descriptions of single controllers were implemented. The logic of the program was optimized to minimize problems during operation. The logic for trips of the cooling water system was reviewed to reduce the risk of trips caused by a malfunction of a sensor. It was tried to evaluate the signals of at least two sensors before a trip of the cooling water system is triggered. If there is no redundant sensor available two related sensors can be evaluated together, e.g. flow rate together with water pressure is used as criteria for a running pump.

About 1200 temperature sensors are mounted at the cryogenic components inside W7-X cryostat. 36 Sensors showed already problems before cool down of OP1.1. These sensors were further checked after OP1.1. The measuring chain, the allocation of the calibration certificates and the input data in the electronic measuring modules were checked. All sorts of errors were found and corrected, so that finally these 36 sensors are operational for OP.1.2.

\section{Structural monitoring of W7-X}

The FE Global Model of the Cryostat System (GMCS) was used to predict and verify the behavior of the cryostat and plasma vessel, their supports and ports during these activities [19]. The GMCS is part of a larger set of FE models used for structural analyses on W7-X components. This set includes the second global model of W7-X which covers the magnet system and its support structure [20]. Both systems can be analyzed independently as there is no relevant mechanical interaction between the two systems.

To verify the structural integrity of the cryostat and magnet system during commissioning and operation several accompanying measurements were prepared [21, 22]. The most relevant load cases for structural monitoring of the cryostat were the evacuation and baking of the vessels. For the super conducting magnet system the cool down process and magnet operation were of main interest. These processes were continuously supervised by mechanical engineers. The measurement data was extracted from the central Data Archive and processed with a special Matlab Tool [23] to enable quick and proper assessment of the structural status of W7-X during commissioning and operation.

The monitoring activities during commissioning of W7-X did not reveal any major structural issues with the plasma vessel and cryostat and their respective supports [24, 25]. The GMCS was used extensively and the instrumentation in form of plasma vessel strain gauges, port displacement sensors and temperature sensors provided a solid basis for comparing the behaviour of the cryostat with the predictions.

With respect to the magnet system results of comparison between numerical modeling and mechanical instrumentation measurements show good agreement after introduction of modifications in the numerical models. The areas within the magnet system FE model that require special attention have been defined based on the first monitoring results. These areas require additional refinement to better match the measurement results. A temperature compensation procedure is developed for the strain gauges on the cold structure and is to be tested in order to be fully functioning during most demanding operation phases. Figure 2 shows a typical example of a monitoring task. Here the radial displacement of the 10 cryoleg lower bearings due to coil energization and subsequent deformation of the central support structure is shown, both the measured values and the predicted values from the magnet system global model.

For the next operational phase OP1.2 (starting mid 2017) the GMCS, magnet system global model and the structural sensors will be used again to support commissioning. Of special interest will be the more energetic plasma pulses (with the integrated input power increased from $4 \mathrm{MJ}$ in OP1.1 to $80 \mathrm{MJ}$ in OP1.2) inducing higher heat loads onto the in-vessel components including the plasma vessel and ports. When adjustment of the plasma vessel is required to reposition the divertor modules a procedure is available to do this. To satisfy physics requirements for the upcoming operation phase OP1.2 it is expected that the set of $2.5 \mathrm{~T}$ magnetic configurations will be extended beyond the reference magnetic configurations as already analyzed in the past years [26]. The approach for fast approval of new magnetic configurations has been developed [26].

\section{ECRH-heating}

During the first experimental campaign of Wendelstein 7-X, the ECRH system, which consisted of 6 gyrotrons with an optical microwave transmission in air, demonstrated a high availability and reliability [27]. Therefore for the second 
campaign, OP 1.2, the system remained essentially unchanged, but was upgraded by 4 further gyrotrons to a total power of about $8 \mathrm{MW}$. In OP1.2 the typical pulse lengths will be extended to $10 \mathrm{~s}$, compared to $1 \mathrm{~s}$ discharges in OP1.1. Even though the whole ECRH system is designed for steady state operation [28], the $10 \mathrm{~s}$ operation exhibits a challenge since it is in the transition between short pulse operation $(<1 \mathrm{~s})$ and stationary operation $(>30 \mathrm{~s})$. Therefore, the electron beam current in the gyrotrons must be stabilized by a feed forward control of the emitter heating, the so called boosting, which has to be developed for each gyrotron individually. The expected higher power density and pulse energy at the transmission line necessitated an improvement of the air quality in the ECRH beam duct with regards to a reduction of humidity and dust. Therefore, the duct was upgraded with an entrance door sluice and air drying system.

The launching system in OP 1.1 consisted of 4 front steering launchers, for 3 beams each (12 beams in total). In Op1.1 each launcher was used at least with one beam. For OP1.2, two additional remote steering launchers (RSL), for one beam each, have been installed [29]. They can be feed with the gyrotrons in the so-called "B position". The B-gyrotron beams can be switched between the front steering and remote steering launchers, which are used for Electron Cyclotron Current Drive (ECCD) and collective Thomson scattering diagnostic (CTS). These remote steering launchers enable beam steering without movable elements in the vacuum vessel (see figure 3).

For machine protection every launcher was also equipped with an immersion tube for video observation for wall protection and inspection. The aspired high density operation at W7-X requires the use of the less absorbing second harmonic ordinary (O2-) mode, which was already successfully demonstrated in OP1.1 at low density. For optimization of the ECRH-absorption 10 reflector tiles with a holographic reflection grating have been installed at the heat shield opposite to the ECRH launchers. These tungsten coated molybdenum tiles will enable an efficient multi pass absorption in the high density operation scenario.

As mentioned above, two ECRH beams will be also used for a collective Thomson scattering diagnostic (CTS), starting in OP 1.2a. The scattered radiation can be collected by a receiving antennas either from the ECRH beam emitted the the RSL launcher or from the beam emitted by the standard ECRH launcher in the bean-shaped cross-section (AEE10, A1). The CTS diagnostics aims at providing non-invasive measurements of the ion temperature profile. The W7-X ECRH gyrotrons emit in the frequency range from 139.9 to $140.2 \mathrm{GHz}$ depending on the gyrotron and requested emission power. The CTS diagnostic is capable of using one of two heating gyrotrons with the emission frequency of $140.1 \mathrm{GHz}$.

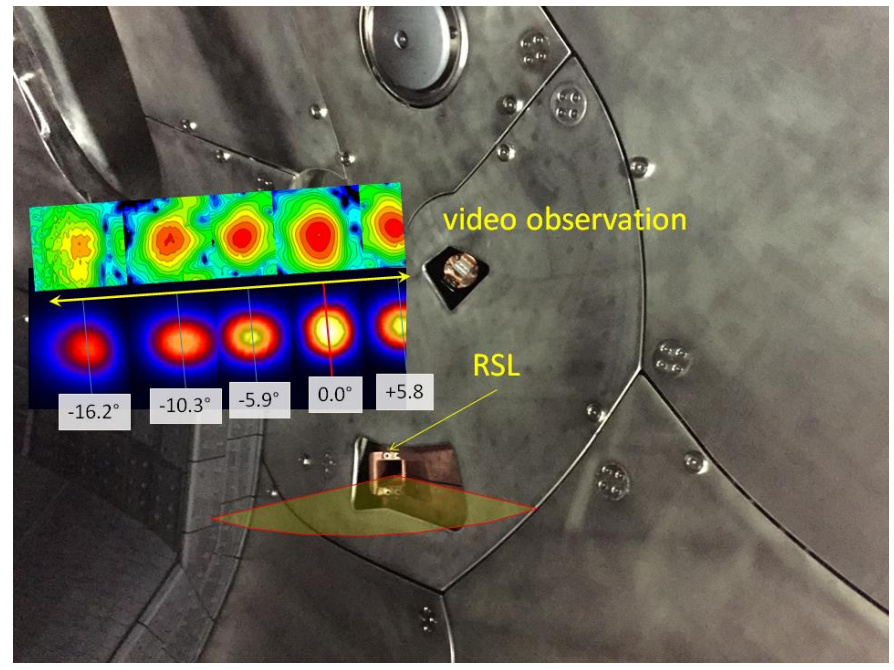

Fig. 3 One of the two, newly installed, Remote Steering Launchers: The front mouth of the launcher with its steering range (red) in the plasma vessel. On the left the calculated (top) and the measured (bottom) beam pattern for a launch angle scan are shown. The pattern was measured at the target close to the plasma axis in front of the launcher inside the plasma vessel. The calculation was done for the same position.

\section{E. Data archiving}

The data collection system for W7-X, named "ArchiveDB", has been developed to collect and archive all technical and experimental data continuously, i.e. $24 \mathrm{hrs}$ a day/7 days a week. The ArchiveDB has been under development since 1998 with the last major architectural changes in 2014 [30]. Since then the ArchiveDB has been up and running without major downtimes.

For the first operation phase the ArchiveDB and its surrounding software components successfully continuously collected and stored the data of machine sensors and all integrated diagnostics. Using a JavaScript Object Notation (JSON)-based interface [31] analysed data and data of diagnostics outside the W7-X standard data acquisition were also successfully stored. This resulted in roughly 35TByte of data for the first operation phase (see figure 4). Browsing and visualizing the data is possible using a standalone tool called 'Databrowser' or via the aforementioned JSON-interface allowing integration into user tools like Matlab or Python-based tools.

For the next operation phase it is planned to support high bandwidth diagnostics and storing data with up to 1GByte/s per signal. Major changes in the underlying hardware and in the software stack are currently being implemented to reach this goal. 


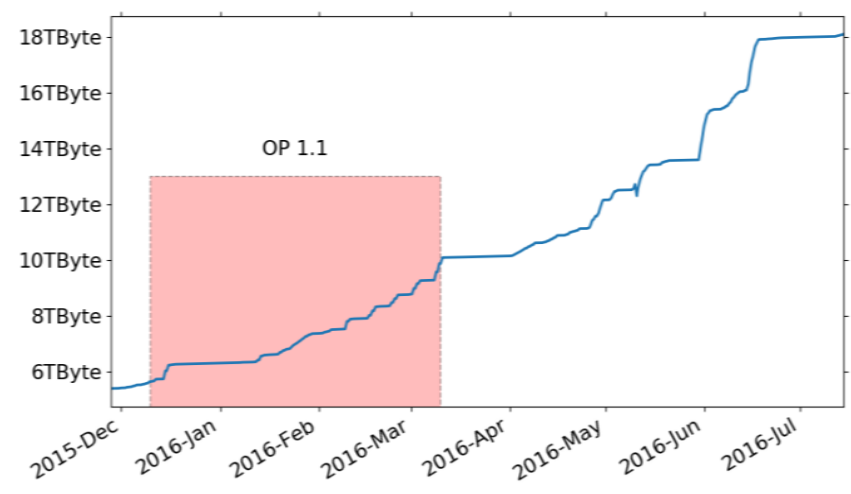

Fig. 4 Data amounts stored in the W7-X ArchiveDB as a function of time. After the end of OP 1.1, up to about July 2016, many evaluated data and raw data acquired with MDS+ and other systems were added to this archive.

\section{F. W7-X device safety and reliability}

Based on the safety analyses and dedicated risk assessments, protection measures for risk mitigation have been defined and implemented in order to ensure a safe design (redundancy, diversity, overpressure protection) as well as an appropriate level of functional safety (central safety systems, safety instrumented functions).

According to the technical standard (International Electrotechnical Commission) IEC 61511, the requirements on safety instrumented functions (SIF) were derived from the safety analysis for the main device as well as from the risk assessments of all associated components and compiled in the safety requirements specification (SRS), which contained 27 personnel related and 9 device safety related SIFs for the operation phase OP1.1.

Residual risks have been reduced by additional organizational safety procedures (access control, safety briefings, operation permits). During the final phase of the assembly extended commissioning work was performed in parallel. During magnetic field operation of super-conducting coils, personnel were not allowed to stay in the Torus hall. However, with ongoing vacuum and cryo supplies, assembly installation and service works including function tests of inactive components inside the Torus hall and the cryostat was carried out. Potential safety risks which may exist when these activities are performed in parallel and the consequently required safety measures have been analysed and implemented. Special attention has been paid to hazards which may result from loss of vacuum, leaking of helium and fire incidents inside the Torus hall.

In order to meet the explosion protection requirements by using hydrogen as the working gas for plasma operation various technical and organizational measures have been implemented. Nitrogen flushing in the inlet side of the rotary vane pumps (TRIVAC D65B) of the plasma vessel vacuum system is used during operation with hydrogen diluting its content in the exhaust gas well below the lower explosion limit. Diagnostics with own vacuum pumps inject nitrogen gas into the exhaust lines to avoid there hydrogen accumulation. All vacuum pumps operated in the torus hall are connected to a central exhaust system certified to meet the ATEX requirements (Ex-Zone 1). Several hydrogen gas sensors as part of the central gas warning system are installed in all areas where a hydrogen gas emission could occur.

A number of global hazard events which could occur on the W7-X stellarator have been analyzed. The way of detecting the events is described as well as the associated technical and control safety measures; administrative procedures and the resulting actions. Systems and components that are important for personnel or public safety are identified considering their functional importance to the overall plant safety. To each hazard event, the corresponding reports and references are given. All control components and systems foreseen for the supervision of W7-X operation have been compiled and assessed regarding their importance for analysis purposes, operation requirements, and for personnel and device safety issues. Based on the analyses, several proposals for design improvements have been issued.

From pumping down the cryostat (July 2014), cooling down (Feb. 2015), and performing the magnetic field tests up to the plasma preparation phase, all systems together with the central safety control system demonstrated a high availability. Technical failures, not unusual during a commissioning phase, could be fixed by the operation team without considerable impact on the project schedule. No quench occurred, but two unplanned fast discharges (2015-09-21, 2016-02-25). No failure of the central safety system has been observed; almost stable operation of all required technical systems during the commissioning phase and plasma experiments 2014-2016 could be achieved.

On September 21st, 2015, the outage of the general (external) power supply of the institute (for about $300 \mathrm{~ms}$ ) during magnetic field tests resulted in a short discharge of the superconducting magnet system. The safety systems, including the emergency power supply, reacted as planned and the W7-X device has been put automatically into the pre-defined safe state. On February 25th, 2016, a fast discharge of the magnet system has been initiated due to an (unexpected) fast rampdown of the trim coils with $20 \mathrm{kA} / \mathrm{s}$. This induced in the nearby located planar coils a voltage, sufficient to trigger the quench detection system.

With 32 scheduled experimental days during OP1.1 (each 10 h) with about 30-50 experimental programs, the device downtime was two days, resulting in a $94 \%$ operational availability. The downtime was caused by a required repair of the glow discharge system (2016-02-11), the mentioned fast discharge of the magnet system (2016-02-25), and a failure of a $20 \mathrm{kV}$ switch of the high voltage supply for the magnet system power supply.

In preparation of OP1.2, the central safety system (cSS) is going to be upgraded by including additional components with their communication interfaces and by implementing new instrumented safety functions (SIF) in order to meet the safety requirements of new process functions such as boronization of the plasma facing components. Furthermore, a Fast Interlock System (FIS) will be put into operation in order to switch the plasma heating systems off within max. $50 \mathrm{~ms}$ in order to avoid overheating and possible damage of in-vessel components in case the plasma performance has been deteriorated and/or the excess of limits (plasma density, plasma radiation) is detected during the plasma operation. 


\section{G. Remote participation}

Research on W7-X is performed by IPP together with European colleagues in the framework of Eurofusion, but the one-team approach also includes colleagues from the US, Japan, Australia and other countries. For this external participation, W7-X follows a remote participation concept, which differs from the concept of a remote control room. Instead, the concept focuses on the scientific working places for being remotely as efficient as on site. The requirements on the remote participation system are therefore to gather information about the planned and running experiments during the experiment day, live signals of the experiment run, access to experiment databases and services, interactively created data of different scientific groups and last but not least the tight communication to the scientific on-site teams.

In collaboration with the Princeton Plasma Physics Laboratory, several systems have been defined, which have been almost completely implemented. The information about the experiment planning is transmitted simply via the web conferencing tool ZOOM, which is also used for the tight communication with the on-site teams. Furthermore, a web based operator logbook is under preparation for a convenient tracing of the operator events and information about the executed experiment run. This system supports already automated tagging regarding e.g. ECRH gyrotron selection, gas feed valves and many more. The live signals are being presented by a web based data monitor, which collects data from the real time plasma control as well as the reduced online data streams of the data acquisition systems and presents the data via a high performance WebGL technique (https://get.webgl.org). Access to the experiment database is enabled by an web based REST API and a direct Java interface, which allows to use the data analysis codes off-site identically as at working places on-site. In parallel, the MDSplus (http://www.mdsplus.org) database has been exposed to the remote access as well. Modelling functions like magnetic field line tracing, 3D MHD equilibria (VMEC) and many more are available through the service oriented architecture with SOAP/XML

(https://www.w3schools.com/xml/xml_soap.asp) and REST APIs. The interactive data will be provided via a NextCloud (https://nextcloud.com) service, which enables to provide file server data through a web interface.

All these services reside as a mirror or proxy system in a so called ExtraNet (according to the standard ISO/IEC 2382), which is a dedicated, non-internal network segment with access as a virtual private network (VPN). The clear separation of this network form internal ones became necessary due to an increased demand for IT security. Although the system has not yet all of the required services, the implementation in the US remote collaboration sites is in progress for an early user feedback.

\section{STATUS OF W7-X FOR THE SECOND EXPERIMENTAL PHASE}

\section{A. In-vessel components}

While in OP 1.1, W7-X was operated with 5 poloidal graphite limiters only [32], before OP 1.2 the first Divertor system was installed, with 10 so-called Temporary Divertor Units (TDU). This is an inertially cooled fine grain graphite divertor with the same divertor geometry and castellation as the planned steady-state Divertor to be installed in 2018-19 [33, 34]. The inertially cooled TDU cannot be damaged by overloading. An overload of the massive divertor tiles only results in carbon blooming. As compared to the limiters, the TDU now can handle about $8 \mathrm{MW} / \mathrm{m}^{2}$ with a cool-down time between pulses of about 20 minutes at the highest energies. The limit of the integrated input power in a discharge is now increased to $80 \mathrm{MJ}$.

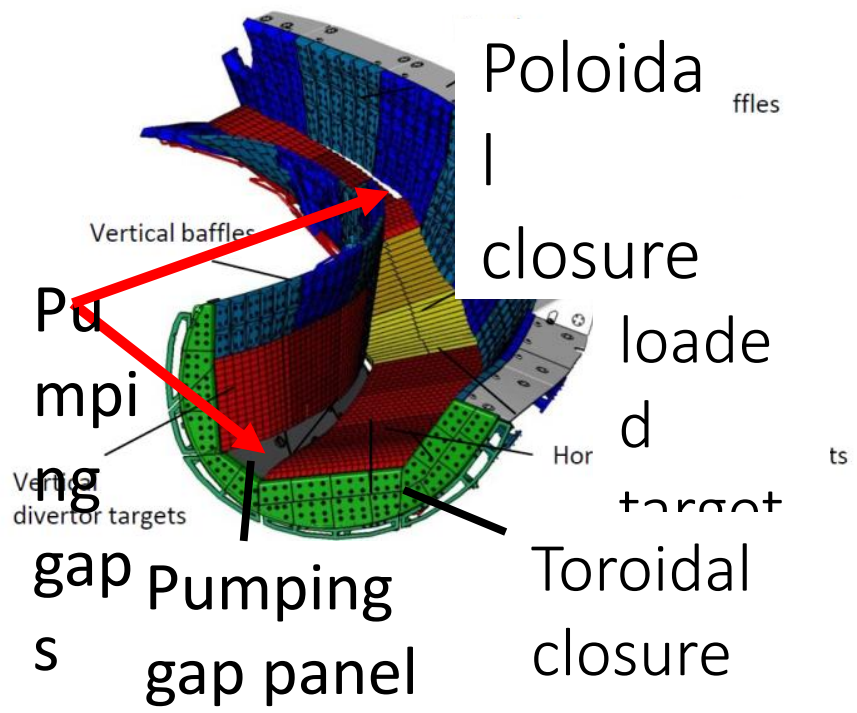

Fig. 5 Schematic view of one of the 10 divertor modules of the Temporary Divertor Unit (TDU), installed in 2016/17. Only the pumping gap panels will be installed after the second operation phase, i.e. in 2018.

Figure 5 shows schematically one of the 10 divertor components with all sub-components [2]. In the TDU, the horizontal and vertical targets are from fine grain graphite. For the stead-state divertor, to be installed 2018-2012, the divertor will be made of CFC on a water-cooled heat sink [33]. All components in this figure are very similar to the later steadystate divertor. This allows using the robust TDU divertor to develop the divertor operation scenarios for the steady-state operation, where the in-vessel components have the same geometry.

During the completion phase after OP 1.1, all 10 TDU modules have been installed in a tight space within the plasma vessel. However, a high accuracy was required to avoid leading edges between the divertor tiles. The required accuracy of 0.2 $\mathrm{mm}$ was not in all cases achieved. A stepsize of up to $0.6 \mathrm{~mm}$ was achieved, which should be tolerable for the TDU. For the stead-state divertor, to be installed 2018-2012, the assembly tools and procedures are being developed in the coming months.

On the plasma vessel walls between the divertor modules, the wall is protected by steel wall panels (already installed before OP 1.1) at the outside, and graphite tiles on $\mathrm{CuCrZr}$ heat sinks at the inner side of the torus. In OP 1.1 the heat sinks were 
already installed, but only in the recent months, the 8000 graphite tiles have been installed. The high accuracy requirements made it necessary to customize about $1 / 3$ of them according to the as-built geometry in the plasma vessel [35].

\section{B. Vacuum and gas-systems, wall-conditioning}

The vacuum pump systems were running already successfully in OP 1.1, but the control systems have been upgraded and also the residual gas analyzers have been incorporated into the data acquisition.

The central gas injection system will be available with full
Boronisation is foreseen to start in the course of OP1.2a. To ensure safe operation only two of ten pumping units of the plasma vessel vacuum system will be used during boronisation, with thermal and chemical decomposers integrated in their exhaust gas line. Furthermore, the gas monitoring system in the torus hall has been upgraded with diborane sensors. Supply, injection and exhaust lines of the hazardous He-diborane gas mixture are strictly separated from those of the standard gas injection and conditioning system. The process gas lines are double walled with the outer tube filled with nitrogen to prevent emission of diborane in case of leakage.

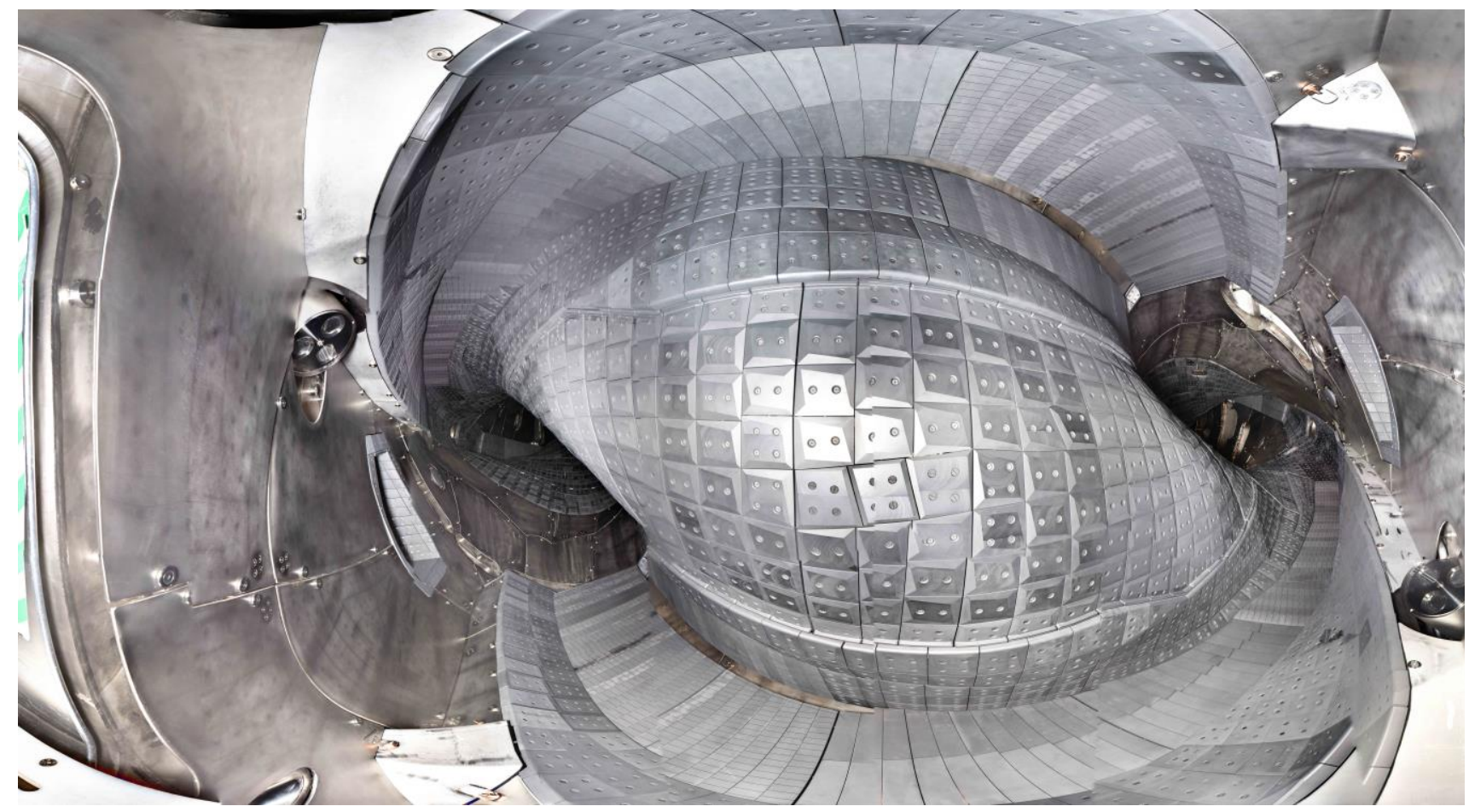

Fig. 6 Fish-Eye view of the W7-X module \#2. Well visible are the divertor module in the bottom and at the top. On the inner side, the graphite tiles can be seen as well as the steel panels on the outside of the torus. Also, the two NBI-ports are visible on both sides, and divertor observation cameras (right bottom and left topside). Image from T. Bräuer.

performance. For plasma fuelling and impurity seeding 10 fast piezo valves are operational at five symmetric inboard positions of the plasma vessel. Both groups can be operated independently with different gases. Generally, the fuelling gas will be hydrogen. Feedback control of the gas flow will be prepared and needs to be commissioned. The line integrated plasma density along a central chord as measured by a dispersion-interferometer will be used as the control parameter.

The mass flow controllers used for glow discharge conditioning have been upgraded before OP 1.2a to provide a higher gas flow. This will allow operating glow discharges at a higher pumping speed of the vacuum system and will improve the efficiency of the cleaning process. For glow discharges all of ten symmetrically distributed electrodes are available, allowing for a current density of up to $15 \mu \mathrm{A} / \mathrm{m}^{2}$ at the first wall.

After baking of the vessel at $150^{\circ} \mathrm{C}$ wall conditioning will be continued with hydrogen glow discharges (order of days) to reduce impurities, followed by short helium glow to remove hydrogen from the first wall.

\section{Plasma heating with NBI and ICRH}

The NBI and ICRH systems are planned to be put into operation in 2018. The NBI system will provide $7 \mathrm{MW}$ of neutral hydrogen injection at $55 \mathrm{keV}$ using two injectors that are each equipped with two beam sources [356. During the slowing down of the NBI produced fast ions, both plasma ions and electrons are being heated. However, some of the fast ions might get lost from the plasma and cause impermissible high heat loads on plasma facing components. Therefore investigations have started to find well suited magnetic configurations and density ranges for operating NBI.

The ICRH system consists of one radially movable double strap antenna and one generator to launch fast waves into the plasma at a power of $0.5 \mathrm{MW}$ to $1 \mathrm{MW}$ depending on the coupling of the antenna [37]. With the available frequencies of the generator the three-ion scheme with a minority of helium 3 is most promising [38]. Since the location of fast ion generation depends only on the location of the appropriate resonance, 
ICRH might become particularly well suited to study the confinement of fast ions.

\section{Diagnostics}

While in OP 1.1 about 20 diagnostic systems have been in operation [39], for OP 1.2, 40 diagnostics systems will be exploited to investigate the plasma parameters, see table 2.

\section{E. Software for the physics experiment programme}

During the first operation phase a complete software tool chain for planning, controlling and monitoring physics experiment programs has been successfully established by the W7-X CoDaC group [40]. Based on the actual operation

\begin{tabular}{|c|lc|c|lc|}
\hline 1 & Spectroscopic and Thermal Imaging & $\mathrm{n}$ & 21 & TESPEL & $\mathrm{b}$ \\
\hline 2 & Scraper Observation & $\mathrm{b}$ & 22 & Zeff & $\mathrm{b}$ \\
\hline 3 & Real-time Image Processing & $\mathrm{n}$ & 23 & Pulse Height Analysis & $\mathrm{i}$ \\
\hline 4 & Divertor Spectroscopy & $\mathrm{n}$ & 24 & XMCTS & $\mathrm{n}$ \\
\hline 5 & UV-Vis-IR Endoscopes & $\mathrm{n}$ & 25 & Magnetics & $\mathrm{i}$ \\
\hline 6 & Helium Beam & $\mathrm{i}$ & 26 & Profile Reflectometer & $\mathrm{n}$ \\
\hline 7 & Filterscope & $\mathrm{i}$ & 27 & Correlation Reflectometry & $\mathrm{i}$ \\
\hline 8 & Coherence Imaging Spectroscopy & $\mathrm{n}$ & 28 & Doppler Reflectometry & $\mathrm{i}$ \\
\hline 9 & Divertor Langmuir Probes & $\mathrm{n}$ & 29 & Poloidal Correlation ECE & $\mathrm{n}$ \\
\hline 10 & Neutral Pressure Gauges & $\mathrm{i}$ & 30 & Radial Correlation ECE & $\mathrm{n}$ \\
\hline 11 & Penning Gauge with Optical Observation & $\mathrm{n}$ & 31 & Phase Contrast Imaging & $\mathrm{n}$ \\
\hline 12 & Plasma-Wall Interaction Probes & $\mathrm{n}$ & 32 & CXRS on NBI & $\mathrm{n}$ \\
\hline 13 & Magnetic Flux Surface Measurement & $\mathrm{i}$ & 33 & Collective Thomson Scattering & $\mathrm{n}$ \\
\hline 14 & Video Diagnostics & $\mathrm{i}$ & 34 & Neutron Monitor & $\mathrm{i}$ \\
\hline 15 & Multi-purpose Manipulator & $\mathrm{I}$ & 35 & Ice Pellet Injection & $\mathrm{n}$ \\
\hline 16 & Alkali Metal Beam & $\mathrm{n}$ & 36 & Fast-ion Diagnostics & $\mathrm{n}$ \\
\hline 17 & Bolometers & $\mathrm{i}$ & 37 & ECE Diagnostics & $\mathrm{i}$ \\
\hline 18 & C/O Monitor & $\mathrm{b}$ & 38 & XICS & $\mathrm{i}$ \\
\hline 19 & HEXOS & $\mathrm{i}$ & 39 & Thomson Scattering & $\mathrm{i}$ \\
\hline 20 & Laser blow-off & $\mathrm{n}$ & 40 & Interferometer & $\mathrm{i}$ \\
\hline
\end{tabular}

TABLE 2: List of the diagnostics system for OP 1.2. The letter on the right side indicates whether a diagnostic system has been improved ("i"), or it's a new system ("n") or if it becomes on-line only later in the second ("b").

experiences and in view of the expected more complex physics programs within the next operation phase, all tools were subjected to a thorough examination. The requirements coming from team working and remote participation (see also section III.G), as well as from general experiment organization had been taken into account. Besides significant enhancements of the existing tools, new developments have been focused on four main work packages:

\section{Automating experiment program categorizing}

The component model framework [41] has been enhanced to automatically retrieve typical experiment program parameters for generating tags describing the intended setup of a program. The resulting tags may be plain labels or may carry a value generated from a customizable evaluation function. Examples are the used gas type or the induced ECRH power in total or per gyrotron. The framework allows applying tags to planned physics programs in the programs database and to the actual running session when executing programs. Additional tags can be added manually from an underlying extendible catalog.

The tagging framework has been fully integrated into the software stack. Thus, tags can be used for categorizing planned physics programs, e.g. for organizing and searching the programs database, or - because program and session tags are logged to the archive - for searching the program logbook.
2. Central electronic logbook for all W7-X team members

An integrated electronic logbook is currently being implemented for common access to both executed physics programs data and operational data or technical events. Session leaders as well as diagnosticians and components' ROs can browse, comment and assess experiment programs or correlate to machine status and technical events. Depending on the user's role, rich text comments and evaluative tags can be added to logs. The logbook provides a fast full text search and quick links to acquired data. It can be accessed via a web browser interface and via a RESTful web service - ready for remote access by any $\mathrm{W} 7-\mathrm{X}$ team member.

\section{Web based live data monitoring}

With focus on the need for remote participation within an international team, the implementation of the live data monitoring has been radically reworked as a web based clientserver system utilizing modern technologies like WebSockets and WebGL for high performance live data visualization [42]. The mouse-centered as well as touch friendly web interface offers support for new and classic access tools: from desktop browsers to tablets or smart phones and the W7-X control room's HiperWall. Data tracks can cover short and long-term (24 hours) monitoring. They can be chosen from presets or intuitively added and configured. Besides scalar time series and 3D profile data, status data on the experiments progress can be visualized.

\section{Customizable live data analysis}

On the way to continuous operation, live data analysis becomes more and more necessary for online evaluation of the experiment's success. Because the evaluating functions themselves are subject of research, a framework has been developed which enables the physicists to provide their own data analysis algorithms. The analysis methods are to be plugged-in into the CoDaStation [43] architecture where they operate on acquired data. The implementation allows cascading analysis methods and combining data from different sources.

\section{SUMMARY}

The first operational phase of W7-X was very successful from the experimental results [27] and also under the technical aspects. Nevertheless, some lessons were learned and resulted in modifications.

In the completion phase after OP 1.1 several components were scheduled to be installed, like the TDU and the graphite tiles in the plasma vessel and the and updates in CoDac and diagnostics systems. In the next experimental phase, also new systems (NI and ICRH) will commence operation. After the end of the in-vessel assembly, re-commissioning has started, and the next operational phase is scheduled to start in August 2017. An inside view of the plasma vessel is shown in fig. 6 .

\section{REFERENCES}

[1] G. Grieger et al., "Physics Optimization of Stellarators", Phys. Fluids B 4 2018-2091 (1992).

[2] H.-S. Bosch et al., "Final integration, commissioning and start of the Wendelstein 7-X stellarator operation", Nucl. Fusion 57, 116015 (2017).

[3] J. Nührenberg et al., Overview on Wendelstein 7-X theory, Trans. Fus. 
Techn. 27, 71-75 (1995).

[4] H.-S. Bosch et al., "Construction of Wendelstein 7-X - Engineering a Steady-State Stellarator", IEEE Transactions Plasma Science 38 (3) 265273 (2010).

[5] T. Braeuer et al., "Interaction of Metrology and Assembly at W7-X", IEEE Transactions on Plasma Science, 42 (7) 1943-1950 (2014).

[6] T. Andreeva et al., "Influence of construction errors on Wendelstein 7-X magnetic configurations", Fusion Eng. Design 84, 408-412 (2009).

[7] T. Andreeva et al., "Evaluation of Wendelstein 7-X magnetic field perturbations during optimized module positioning", Proc. 39th EPS Conference, Stockholm, Sweden, (2012).

[8] T. Andreeva et al., "Tracking of the magnet system geometry during Wendelstein 7-X construction to achieve the designed magnetic field", Nuclear Fusion, 55 (6) 063025 (2015).

[9] T. Andreeva et al., "Estimation of Wendelstein 7-X magnetic field perturbation due to permeable materials", Proc. 42th EPS Conference, Lisbon, Portugal, (2015).

[10] V. Bykov et al., "Structural analysis of W7-X: From design to assembly and operation", Fusion Eng. Design 86 (6-8) 645-650 (2011).

[11] V. Bykov et al., "Specific Features of Wendelstein 7-X Structural Analyses", IEEE Trans. Plasma Science 42 (3) 690-697 (2014).

[12] J. Fellinger, et al., "Asymmetry of W7-X magnet system introduced by torus assembly", Fusion Eng. Design, 89, 2189-2193 (2014).

[13] K. Riße, et al., "Wendelstein 7-X-Commissioning of the Superconducting Magnet System", IEEE Trans. on Appl. Supercond. 26, 4202004 (2016).

[14] T. Rummel, et al., "Commissioning Results of the Super-conducting Magnet System of Wendelstein 7-X”, IEEE Trans. Appl. Supercond. 27, 1-7 (2017).

[15] T. Rummel et al., "The trim coils for the Wendelstein 7-X magnet system", IEEE Trans. Appl. Supercond., 22, 4201704 (2012).

[16] F. Füllenbach et al., "The Wendelstein 7-X Trim Coil sytem commissioning and first operational results", Fusion Eng. Design, 124 94-98 (2017).

[17] M. Nagel, et al., "Cryogenic commissioning, cool down and first magnet operation of Wendelstein 7-X," IOP Conf. Series: Materials Science and Engineering 171, 012050 (2017).

[18] M. Nagel, et al., "First evaluation of cryogenic performance of Wendelstein 7-X cryostatt", Fusion Eng. Design, 123, 153-157 (2017).

[19] C.P. Dhard, et al., "Refrigerator operation during commissioning and first plasma operations of Wendelstein 7-X”, Fusion Eng. Design, 123 111-114 (2017).

[20] P. van Eeten et al., "Features and analyses of W7-X cryostat system FE model”, Fusion Eng. Design, 96-97 369-372 (2015).

[21] V. Bykov et al., "Engineering Challenges of W7-X: Improvement of Numerical Modelling and Mechanical Monitoring after Commissioning and First Phase of Operation", Fusion Sci. Tech., 72, 546-558 (2017).

[22] V. Bykov et al., "Wendelstein 7-X mechanical instrumentation system for commissioning and operation", Fusion Sci. Tech., 68 267-271 (2015).

[23] A. Carls et al., "A structural integrity monitoring tool for Wendelstein 7$\mathrm{X}$ ", to be published in Fus. Eng. Design.

[24] H.-S. Bosch et al., "Experience with the commissioning of the superconducting stellarator Wendelstein 7-X", Fusion Eng. Design, 9697 22-27 (2015).

[25] P. van Eeten et al., "Monitoring of W7-X Cryostat Commissioning with Cryostat System FE Model”, Fus. Eng. Design, 123, 91-96 (2017).

[26] V. Bykov et al., "Mechanical Monitoring Issues in Preparation to Next Step of W7-X Operation", IEEE Trans. Plasma Science in print, DOI: 10.1109/TPS.2017.2786744.

[27] R. Wolf et al., "Major results from the first plasma campaign of the Wendelstein 7-X stellarator", Nucl. Fusion, 57, 102020 (2017).

[28] V. Erckmann et al., "Electron Cyclotron Heating for W7-X: Physics and Technology", Fusion Science and Technology 52, 291-312 (2007).

[29] C. Lechte et al., "Remote-steering launchers for the ECRH system on the stellarator W7-X," 2013 38th International Conference on Infrared, Millimeter, and Terahertz Waves (IRMMW-THz), Mainz, 2013, pp. 1-2. doi: 10.1109/IRMMW-THz.2013.6665754.

[30] C. Henning et al., "ArchiveDB - Scientific and technical data archive for W7-X”, Fusion Eng. Design., 112, 984-990 (2016).

[31] M. Grahl et al., "Archive WEBAPI: a webservice for the experiment data archive of Wendelstein 7-X", Fusion Eng. Des., in print.

[32] T. S. Pedersen, et al., "Plans for the first plasma operation of Wendelstein 7-X”, Nuclear Fusion, 55, 126001 (2015).

[33] H. Renner, et al., "The capabilities of steady state operation at the stellarator W7-X with emphasis on divertor design”, Nuclear Fusion 40 (6) 263-1093 (2000).

[34] R. Stadler et al., "The in-vessel components of the experiment Wendelstein 7-X", Fusion Eng. Design, 84 (2-6) 305-308 (2009).

[35] T. Braeuer et al., "Customized Manufacture of Graphite Tiles According to the As-Built-Situation of Wendelstein 7-X Interior", presented at 13. ISFNT, 2017.

[36] P. McNeely et al., "Current status of the neutral beam heating system of W7-X”, Fusion Eng. Design 881034 (2013).

[37] J. Ongena et al., "Study and design of the ion cyclotron resonance heating system for the stellarator Wendelstein 7-X", Phys. Plasmas 21061514 (2014).

[38] Ye. O. Kazakov et al., "Efficient generation of energetic ions in multiion plasmas by radio-frequency heating“, Nat. Physics (2017).

[39] R. König, et al., "The Set of Diagnostics for the First Operation Campaign of the Wendelstein 7-X Stellarator", Jour. Instrumentation 10 P10002 (2015).

[40] A.Spring et al., "Establishing the Wendelstein 7-X steady state control and data acquisition system during the first operation phase", Fusion Eng. Design, 123 579-583 (2017).

[41] M. Lewerentz et al., "Experiment planning using high-level component models at W7-X", Fusion Eng. Design 87 1949-1953 (2012).

[42] S. Dumke et al. ,Next generation web based live data monitoring for W7X", Fusion Eng. Design, 129, 16-23 (2018).

[43] T. Bluhm et al., "Wendelstein 7-X's CoDaStation: A modular application for scientific data acquisition”, Fusion Eng. Design 89 658-662 (2014). 